\title{
Absolute Extensors and Binary Monads
}

\author{
T. Radul ${ }^{1,2}$
}

Received: 4 September 2014 / Accepted: 7 March 2016 / Published online: 15 March 2016

(C) The Author(s) 2016. This article is published with open access at Springerlink.com

\begin{abstract}
Let $\mathbb{F}$ be a monad in the category Comp of compact Hausdorff spaces and continuous maps. An abstract convexity was constructed by Radul for each $\mathbb{F}$-algebra of the monad $\mathbb{F}$ in the category Comp. It was proved that if the convexity of the monad $\mathbb{F}$ with some additional properties is binary then $\mathbb{F}$ has good topological properties, in particular, $F X$ is an absolute extensor in the class of 0 -dimensional spaces for each openly generated compactum $X$. We show in this paper that binarity is also a necessary condition.
\end{abstract}

Keywords Monad - Convexity · Binarity - Absolute extensor in the class of 0-dimensional compacta

Mathematics Subject Classification (2010) $18 \mathrm{~B} 30 \cdot 18 \mathrm{C} 15 \cdot 52 \mathrm{~A} 01 \cdot 54 \mathrm{~B} 30$

\section{1}

The notion of convexity considered in this paper is considerably broader than the classical one; specifically, it is not restricted to the context of linear spaces. Such convexities appeared in the process of studying different structures like partially ordered sets, semilattices, lattices, superextensions etc. We base our approach on the notion of topological convexity of [21] where the general convexity theory is covered from axioms to applications in different areas.

T. Radul

tarasradul@yahoo.co.uk

1 Institute of Mathematics, Casimirus the Great University, Bydgoszcz, Poland

2 Department of Mechanics and Mathematics, Lviv National University, Universytetska st.,1, 79000 Lviv, Ukraine 
In [13], a certain categorical connection between topological-algebraic structures and convexities was found. Many topological constructions are functorial; they are defined not only for spaces but for maps as well. Certain functors in categories of topological spaces and continuous maps have also natural algebraical structures. Such structures can be described by the notion of monad (or triple) structure in the sense of S.Eilenberg and J.Moore and their corresponding category of Eilenberg-Moore algebras [3].

Many classical constructions lead to monads: hyperspaces, spaces of probability measures, superextensions etc. (See for example the survey [12] or the book [18]). A number of authors consider certain convexity structures to investigate monads. The linear convexity corresponds to the probability measure monad [17]. Certain non-linear convexities correspond to the superextension monad [7], the inclusion hyperspace monad [16] and the idempotent measure monad [22]. A convexity structure on each $\mathbb{F}$-algebra for any monad $\mathbb{F}$ in the category of compact Hausdorff spaces and continuous maps was introduced in [13]. These convexities are preserved by $\mathbb{F}$-algebra morphisms. Let us remark that our general construction of convexities includes the above mentioned convexities for probability measures, superextension, hyperspaces of inclusion and idempotent measures.

Let us consider two classical theorems about topological properties of the probability measure functor $P$ and the superextension functor $\lambda$.

Theorem 1.1 (Ditor, Haydon [1]) For a compactum $X$ the space of probability measures $P X$ is an absolute retract if and only if $X$ is an openly generated compactum with weight $\leq \omega_{1}$.

Let us remark that in [1] the above theorem was formulated for Dugundji spaces but it is known that each openly generated compactum of weight $\leq \omega_{1}$ is a Dugundji space [20, Th. 5].

Theorem 1.2 (Ivanov [8]) For a compactum $X$ the superextension $\lambda X$ is an absolute retract if and only if $X$ is an openly generated continuum.

The notion of absolute retract (shortly AR) coincides with the notion of absolute extensor (shortly AE) in the class of compacta (see for example [11]). The notion of absolute extensor in the class of 0 -dimensional compacta (shortly $\mathrm{AE}(0)$ ) is considerably broader than the notion of absolute extensor. For example, the Cantor cube $D^{\tau}$ is $\mathrm{AE}(0)$ but certainly not AE. However, these notions are equivalent in the realm of linear convexity. Each (linearly) convex compactum is $\mathrm{AE}$ if and only if it is $\mathrm{AE}(0)$ [5]. Since the compactum $P X$ is convex, we have that $P X$ is not even $\mathrm{AE}(0)$ for any compactum $X$ with weight $\geq \omega_{2}$. But we have that $\lambda X$ is $\mathrm{AE}(0)$ for each openly generated compactum $X$ [9]. This difference between properties of functors one can explain in terms of properties of the corresponding convexities. The convexity generated by the superextension has the binarity property, which the linear convexity does not have. It was shown in [13] that each monad which generates a binary convexity has good topological properties. More precisely, Theorem 3.3 of [13] states that for each binary L-monad $\mathbb{F}$ which weakly preserves preimages (we also assume that the functorial part $F$ of the monad $\mathbb{F}$ preserves monomorphisms, intersections and one-point spaces) and for each compactum $X$ such that $F X$ is an openly generated compactum, each map $f: F X \rightarrow Y$ with $\mathbb{F}$-convex fibers is 0 -soft provided $f$ is open. If we put $Y=\{*\}$ we obtain that for each binary L-monad $\mathbb{F}$ which weakly preserves preimages $F X$ is $\operatorname{AE}(0)$ provided $F X$ to be an openly generated compactum. Let us remark that it is enough for $X$ 
to be openly generated compactum in case when the functor $F$ is open (i.e. preserves open maps).

The main aim of this paper is to show that the binarity property is also necessary: if $F P D^{\omega_{2}}$ is $\mathrm{AE}(0)$ (for a monad $\mathbb{F}$ with some additional natural properties) then the corresponding convexity is binary (where $P D^{\omega_{2}}$ is the space of probability measures of the Cantor cube of the weight $\omega_{2}$ ). Let us remark that $P D^{\omega_{2}}$ is an openly generated compactum ( $P$ is an open functor [2]) which is not $\mathrm{AE}(0)$ by Theorem 1.1 .

The paper is organized as follows. In Section 2 we recall necessary definitions and the construction of an abstract convexity for a monad of [13]. In Section 3 we obtain a certain sufficient condition for a monad to be binary and in Section 4 we obtain the main result.

By Comp we denote the category of compact Hausdorff spaces (compacta) and continuous maps. Let $X$ be a compactum. We denote by $C(X)$ the Banach space of all continuous functions $\varphi: X \rightarrow \mathbb{R}$ with the usual sup-norm: $\|\varphi\|=\sup \{|\varphi(x)| \mid x \in X\}$. In what follows, all spaces and maps are assumed to be in Comp except for $\mathbb{R}$ and maps in sets $C(X)$ with $X$ compact Hausdorff.

The family $\mathcal{C}$ of closed subsets of $X$ is called a convexity on $X$ if $\mathcal{C}$ is stable under intersection and contains $X$ and the empty set. The elements of $\mathcal{C}$ are called $\mathcal{C}$-convex. Let us remark that we modify the definition of [21]. We consider only closed convex sets. A convexity in the sense of [21] can be obtained as the collection of all unions of up-directed families of sets in $\mathcal{C}$.

We need some definitions concerning monads and algebras (see [10] for more details). We define them only for the category Comp.

A monad $\mathbb{T}=(T, \eta, \mu)$ in the category Comp consists of an endofunctor $T:$ Comp $\rightarrow$ Comp and natural transformations $\eta: \operatorname{Id}_{\text {Comp }} \rightarrow T$ (unity), $\mu: T^{2} \rightarrow T$ (multiplication) satisfying the relations $\mu \circ T \eta=\mu \circ \eta T=\mathbf{1}_{T}$ and $\mu \circ \mu T=\mu \circ T \mu$. (By $\operatorname{Id}_{\text {Comp }}$ we denote the identity functor on the category Comp and $T^{2}$ is the superposition $T \circ T$ of $T$.)

Let $\mathbb{T}=(T, \eta, \mu)$ be a monad in the category Comp. A pair $(X, \xi)$, where $\xi: T X \rightarrow X$ is continuous map, is called a $\mathbb{T}$ - algebra if $\xi \circ \eta X=i d_{X}$ and $\xi \circ \mu X=\xi \circ T \xi$.

Let $(X, \xi)$ be an $\mathbb{F}$-algebra for a monad $\mathbb{F}=(F, \eta, \mu)$ and $A$ be a non-empty closed subset of $X$. Denote by $f_{A}$ the quotient map $f_{A}: X \rightarrow X / A$ (the classes of equivalence are the one-point sets $\{x\}$ for $x \in X \backslash A$ and the set $A$ ) and put $a=f_{A}(A)$. Denote $A^{+}=\left(F f_{A}\right)^{-1}(\eta(X / A)(a))$. (Let us remark that the set $A^{+} \subset F X$ could be define for each closed subset $A$ of any compactum $X$, without additional requirement on $X$ to be an $\mathbb{F}$ algebra). It is easy to check that $A^{+} \subset B^{+}$for each pair of closed subsets of $X$ with $A \subset B$. Define the $\mathbb{F}$-convex hull $C_{\mathbb{F}}(A)$ of $A$ by $C_{\mathbb{F}}(A)=\xi\left(A^{+}\right)$. Put additionally $C_{\mathbb{F}}(\emptyset)=\emptyset$. We define the family $\mathcal{C}_{\mathbb{F}}(X, \xi)=\left\{A \subset X \mid A\right.$ is closed and $\left.\mathcal{C}_{\mathbb{F}}(A)=A\right\}$. The elements of the family $\mathcal{C}_{\mathbb{F}}(X, \xi)$ are called $\mathbb{F}$-convex. It was shown in [13] that the family $\mathcal{C}_{\mathbb{F}}(X, \xi)$ forms a convexity on $X$.

We recall and investigate the property of binarity for a monad. Let $\mathcal{S}$ be a family of subsets of a set $X$. We say that $\mathcal{S}$ is linked if the intersection of each pair of elements of $\mathcal{S}$ is nonempty. $\mathcal{S}$ is called binary if the intersection of every linked subsystem is non-empty. We call 
a monad $\mathbb{F}$ binary if $\mathcal{C}_{\mathbb{F}}(X, \xi)$ is binary for each $\mathbb{F}$-algebra $(X, \xi)$. We shall find a certain sufficient condition for a monad $\mathbb{F}=(F, \eta, \mu)$ to be binary.

Proposition 3.1 Let $\mathcal{S}$ be a closed under intersection family of subsets of a set $X$ such that each linked subfamily consisting of 3 elements has non-empty intersection. Then each finite linked subfamily of $\mathcal{S}$ has non-empty intersection.

Proof Let $\mathcal{C}$ be a finite linked subfamily of $\mathcal{S}$. Let us use induction on cardinality of $\mathcal{C}$, starting from 3. For $|\mathcal{C}|=3$ the statement is trivial. Assume that our statement is true for each subfamily $\mathcal{C}$ with $|\mathcal{C}| \leq n \geq 3$. Consider a linked subfamily $\mathcal{C}=\left\{C_{0}, C_{1}, \ldots, C_{n}\right\}$ and define a family $\mathcal{C}^{\prime}=\left\{C_{0} \cap C_{1}, \ldots, C_{0} \cap C_{n}\right\}$. Since each 3-element subfamily of $\mathcal{C}$ has non-empty intersection, we have that the family $\mathcal{C}^{\prime}$ is linked, hence it has non-empty intersection by induction assumption. But $\cap \mathcal{C}=\cap \mathcal{C}^{\prime}$ and the proposition is proved.

Lemma 3.2 Let $f: X \rightarrow Y$ be a continuous map. Then $F f\left(B^{+}\right) \subset(f(B))^{+}$for each closed subset $B \subset X$.

Proof Let $\chi_{B}: X \rightarrow X / B$ be the quotient map. Put $b=\chi_{B}(B)$. Let $\chi_{f(B)}: Y \rightarrow Y / f(B)$ be the quotient map and put $b^{\prime}=\chi_{f(B)}(f(B))$. There exists a unique map $f^{\prime}: X / B \rightarrow$ $Y / f(B)$ such that $f^{\prime} \circ \chi_{B}=\chi_{f(B)} \circ f$. Let us remark that the map $f^{\prime}$ is continuous and $f^{\prime}(b)=b^{\prime}$. Consider any $v \in F f\left(B^{+}\right)$. Then there exists $\mu \in B^{+}$such that $F f(\mu)=v$. Since $\mu \in B^{+}$, we have $F\left(\chi_{B}\right)(\mu)=\eta(X / B)(b)$. Since $\eta$ is a natural transformation, we have $F f^{\prime} \circ F\left(\chi_{B}\right)(\mu)=\eta(Y / f(B))\left(b^{\prime}\right)$. On the other hand $F f^{\prime} \circ F\left(\chi_{B}\right)=F\left(\chi_{f(B)}\right) \circ F f$, hence $F\left(\chi_{f(B)}\right)(v)=\eta(Y / f(B))\left(b^{\prime}\right)$ and $v \in(f(B))^{+}$.

We say that a functor $F$ preserves one-point preimages iff for each continuous map $h$ : $X \rightarrow Y$ and each $y \in Y$ with $h^{-1}(y)=\{x\}$ we have $(F h)^{-1}(\eta Y(y))=\{\eta X(x)\}$.

Lemma 3.3 Let $f: X \rightarrow Y$ be a continuous map and a functor $F$ preserves one-point preimages. Then we have $(F f)^{-1}\left(A^{+}\right)=\left(f^{-1}(A)\right)^{+}$for each closed $A \subset Y$ such that $f^{-1}(A) \neq \emptyset$.

Proof Let $\chi_{A}: Y \rightarrow Y / A$ be the quotient map. Put $a=\chi_{A}(A)$. Let $\chi_{f^{-1}(A)}: X \rightarrow$ $X / f^{-1}(A)$ be the quotient map and put $a^{\prime}=\chi_{f^{-1}(A)}\left(f^{-1}(A)\right)$. There exists a unique map $f^{\prime}: X / f^{-1}(A) \rightarrow Y / A$ such that $f^{\prime} \circ \chi_{f^{-1}(A)}=\chi_{A} \circ f$. Let us remark that the map $f^{\prime}$ is continuous and $\left(f^{\prime}\right)^{-1}(a)=\left\{a^{\prime}\right\}$.

Consider any $v \in\left(f^{-1}(A)\right)^{+}$. Then we have $F\left(\chi_{f^{-1}(A)}\right)(v)=\eta\left(X / f^{-1}(A)\left(a^{\prime}\right)\right.$. Since $\eta$ is a natural transformation, we have $F f^{\prime} \circ F\left(\chi_{f^{-1}(A)}\right)(v)=\eta(Y / A)(a)$. Since $F f^{\prime} \circ$ $F\left(\chi_{f^{-1}(A)}\right)=F\left(\chi_{A}\right) \circ F f$, we have $F f(v) \in A^{+}$, hence $v \in(F f)^{-1}\left(A^{+}\right)$.

Now let $v \in(F f)^{-1}\left(A^{+}\right)$. Then we have $F f^{\prime} \circ F\left(\chi_{f^{-1}(A)}\right)(v)=F\left(\chi_{A}\right) \circ F f(v)=$ $\eta(Y / A)(a)$. Since $F$ preserves one-point preimages and $\left(f^{\prime}\right)^{-1}(a)=\left\{a^{\prime}\right\}$, we have $F\left(\chi_{f^{-1}(A)}\right)(v)=\eta\left(X / f^{-1}(A)\left(a^{\prime}\right)\right.$, hence $v \in\left(f^{-1}(A)\right)^{+}$.

It is easy to see that $\{a\}^{+}=\{\eta Y(a)\}$ for each $a \in Y$. Hence we obtain the following corollary considering one-point set in the previous lemma. 
Corollary 3.4 Let $f: X \rightarrow Y$ be a continuous map and a functor $F$ preserves onepoint preimages. Then we have $(F f)^{-1}(\eta Y(a))=\left(f^{-1}(a)\right)^{+}$for each $a \in Y$ such that $f^{-1}(a) \neq \emptyset$.

A compactum $X$ is called 0-dimensional if $X$ has a base consisting of open-and-closed sets. For each finite open cover $\left\{U_{1}, \ldots, U_{n}\right\}$ of a 0 -dimensional compactum $X$ there exists a finite open cover $\left\{V_{1}, \ldots, V_{n}\right\}$ such that $V_{i} \subset U_{i}$ for each $i \in\{1, \ldots, n\}$ and $V_{i} \cap V_{j}=\emptyset$ provided $i \neq j$. Let us remark that sets $V_{i}$ are closed as well. (See [4] for more information about 0 -dimensional spaces).

Let us remark that the epimorphisms in the category Comp are exactly the continuous surjections.

Theorem 3.5 Let $\mathbb{F}=(F, \eta, \mu)$ be a monad such that $F$ preserves epimorphisms and onepoint preimages. If there exist a compactum $X$ and a linked system $\left\{A_{1}, A_{2}, A_{3}\right\}$ of closed subsets of $X$ such that $\bigcap_{i=1}^{3} A_{i}=\emptyset$ and $\bigcap_{i=1}^{3} A_{i}^{+} \neq \emptyset$, then $\mathbb{F}$ is binary.

Proof Consider any $\mathbb{F}$-algebra $(Y, \xi)$ and a linked family $\mathcal{B} \subset \mathcal{C}_{\mathbb{F}}(Y, \xi)$. We will show that $\cap \mathcal{B} \neq \emptyset$. Since $Y$ is compact, it is enough to consider $\mathcal{B}=\left\{B_{1}, B_{2}, B_{3}\right\}$ by Proposition 3.1. We can choose a 0 -dimensional compactum $Z$ and a continuous surjective map $h: Z \rightarrow X$. Then the family $\left\{h^{-1}\left(A_{i}\right) \mid i \in\{1,2,3\}\right\}$ is a linked family with empty intersection. Since the compactum $Z$ is 0 -dimensional, we can write $Z=\bigcup_{i=1}^{3} V_{i}$ where the sets $V_{i}$ are closed, pairwise disjoint, $V_{i} \supset \bigcap_{j \in\{1,2,3\} \backslash\{i\}} h^{-1}\left(A_{j}\right)$ and $V_{i} \cap h^{-1}\left(A_{i}\right)=\emptyset$.

We can choose a continuous map $f: Z \rightarrow Y$ such that $f\left(V_{i}\right) \subset \bigcap_{j \in\{1,2,3\} \backslash\{i\}} B_{j}$ for each $i \in\{1,2,3\}$. Since $\bigcap_{i=1}^{3} A_{i}^{+} \neq \varnothing$ and $F$ preserves epimorphisms, there exists $\alpha \in \bigcap_{i=1}^{3}(F h)^{-1}\left(A_{i}^{+}\right)$. We have $\bigcap_{i=1}^{3}(F h)^{-1}\left(A_{i}^{+}\right)=\bigcap_{i=1}^{3}\left(h^{-1}\left(A_{i}\right)\right)^{+}$by Lemma 3.3. Since $h^{-1}\left(A_{i}\right) \subset \bigcup_{j \in\{1,2,3\} \backslash\{i\}} V_{j}$, we have $f\left(h^{-1}\left(A_{i}\right)\right) \subset \bigcup_{j \in\{1,2,3\} \backslash\{i\}} f\left(V_{j}\right) \subset$ $\bigcup_{j \in\{1,2,3\} \backslash\{i\}} \bigcap_{k \in\{1,2,3\} \backslash\{j\}} B_{k} \subset B_{i}$ for each $i \in\{1,2,3\}$. Then we obtain that $F f(\alpha) \in F f\left(\bigcap_{i=1}^{3}\left(h^{-1}\left(A_{i}\right)\right)^{+}\right) \subset \bigcap_{i=1}^{3} F f\left(\left(h^{-1}\left(A_{i}\right)\right)^{+}\right) \subset($ by Lemma 3.2$) \subset$ $\bigcap_{i=1}^{3}\left(f\left(h^{-1}\left(A_{i}\right)\right)\right)^{+} \subset \bigcap_{i=1}^{3} B_{i}^{+}$, hence $\bigcap_{i=1}^{3} B_{i}^{+} \neq \emptyset$. Then we have $\bigcap_{i=1}^{3} B_{i}=$ $\bigcap_{i=1}^{3} \xi\left(B_{i}^{+}\right) \neq \emptyset$.

The class of $L$-monads was introduced in [15] under another name and it contains many well-known monads in Comp like superextension, hyperspace, probability measures etc (see also [14] for a similar notion). For $\varphi \in C(X)$ by $\max \varphi(\min \varphi)$ we denote $\max _{x \in X} \varphi(x)\left(\min _{x \in X} \varphi(x)\right)$ and by $\pi_{\varphi}$ or $\pi(\varphi)$ we denote the corresponding projection $\pi_{\varphi}: \prod_{\psi \in C(X)}[\min \psi, \max \psi] \rightarrow[\min \varphi, \max \varphi]$. It was shown in [15] that for each L-monad $\mathbb{F}=(F, \eta, \mu)$ one can consider $F X$ as a subset of the product $\prod_{\varphi \in C(X)}[\min \varphi, \max \varphi]$, moreover, we have $\pi_{\varphi} \circ \eta X=\varphi, \pi_{\varphi} \circ \mu X=\pi\left(\pi_{\varphi}\right)$ for all $\varphi \in C(X)$ and $\pi_{\psi} \circ F f=\pi_{\psi \circ f}$ for all $\psi \in C(Y), f: X \rightarrow Y$. We could consider these properties of $L$-monads as a definition [15].

What follows we shall assume that each monad is an $L$-monad with some fixed inclusion $F X \subset \prod_{\varphi \in C(X)}[\min \varphi, \max \varphi]$. We will use a simpler notation $v(\varphi)=\pi_{\varphi}(v)$ for $v \in F X$ and $\varphi \in C(X)$. It is easy to check that for each closed subset $A \subset X$ we have $A^{+}=\{v \in$ $F X \mid v(\varphi)=c$ for each $\varphi \in C(X)$ such that $\varphi \mid A \equiv c$ for some $c \in \mathbb{R}\}$. Then the family 
$\mathcal{O}=\left\{O\left\langle\varphi_{1}, \ldots, \varphi_{k} ; c_{1}, \ldots, c_{k} ; \varepsilon\right\rangle \mid k \in \mathbb{N}, \varphi_{i} \in C(X), c_{i} \in \mathbb{R}\right.$ such that $\varphi_{i} \mid A \equiv c_{i}$ and $\varepsilon>0\}$ forms a base of neighborhoods of $A^{+}$, where $O\left\langle\varphi_{1}, \ldots, \varphi_{k} ; c_{1}, \ldots, c_{k} ; \varepsilon\right\rangle=\{v \in$ $F X|| v\left(\varphi_{i}\right)-c_{i} \mid<\varepsilon$ for each $\left.i \in\{1, \ldots, k\}\right\}$. Indeed, consider a closed subset $B \subset F X$ such that $B \cap A^{+}=\emptyset$. Then for each point $\beta \in B$ there exist $\varphi_{\beta} \in C(X), \varepsilon_{\beta}>0$ and $c_{\beta} \in \mathbb{R}$ such that $\varphi_{\beta} \mid A \equiv c_{\beta}$ and $\left|\beta\left(\varphi_{\beta}\right)-c_{\beta}\right|>\varepsilon_{\beta}$. Put $V_{\beta}=\left\{v \in F X|| v\left(\varphi_{\beta}\right)-c_{\beta} \mid>\varepsilon_{\beta}\right\}$. Then $V_{\beta}$ is an open subset of $F X$ such that $\beta \in V_{\beta} \subset F X \backslash A^{+}$. Since $F X$ is compact, we can choose $\beta_{1}, \ldots, \beta_{k} \in B$ such that $\cup_{i \in\{1, \ldots, k\}} V_{\beta_{i}} \supset B$. Put $\varepsilon=\min \left\{\varepsilon_{\beta_{i}} \mid i \in\{1, \ldots, k\}\right\}$. Then $A^{+} \subset O\left\langle\varphi_{\beta_{1}}, \ldots, \varphi_{\beta_{k}} ; c_{\beta_{1}}, \ldots, c_{\beta_{k}} ; \varepsilon\right\rangle \subset F X \backslash B$.

Lemma 4.1 Let $\mathbb{F}=(F, \eta, \mu)$ be an L-monad which is not binary and such that $F$ preserves epimorphisms and one-point preimages. Let $X$ be a compactum, $C$ be a non-empty closed subset of $X$ and $U$ be an open neighborhood of $C$. Let $\left\{\left(A_{\alpha} ; B_{\alpha}\right) \mid \alpha \in \Lambda\right\}$ be a family of pairs of closed subsets of $X$ such that $\emptyset \neq A_{\alpha} \cap B_{\alpha} \subset X \backslash U$ for each $\alpha \in \Lambda$. Then there exists an open subset $V \subset F X$ such that $C^{+} \subset V$ and $A_{\alpha}^{+} \cap B_{\alpha}^{+} \subset F X \backslash V$ for each $\alpha \in \Lambda$.

Proof Choose a 0-dimensional compactum $Y$ and a surjective map $f: Y \rightarrow X$. Since $Y$ is 0 -dimensional, we can choose for each $\alpha \in \Lambda$ non-empty closed, pairwise disjoint subsets $Y_{1}, Y_{2}^{\alpha}$ and $Y_{3}^{\alpha}$ of $Y\left(Y_{1}\right.$ does not depend on $\left.\alpha\right)$ such that $Y \backslash f^{-1}(C) \supset Y_{1} \supset f^{-1}(X \backslash U)$, $Y_{2}^{\alpha} \supset f^{-1}\left(A_{\alpha}\right) \backslash Y_{1}, Y_{3}^{\alpha} \supset f^{-1}\left(B_{\alpha}\right) \backslash Y_{1}$ and $Y_{1} \cup Y_{2}^{\alpha} \cup Y_{3}^{\alpha}=Y$. For $\alpha \in \Lambda$ consider the continuous map $h_{\alpha}: Y \rightarrow\{1,2,3\}$ defined as follows: $h_{\alpha}\left(Y_{1}\right)=\{1\}, h_{\alpha}\left(Y_{2}^{\alpha}\right)=\{2\}$ and $h_{\alpha}\left(Y_{3}^{\alpha}\right)=\{3\}$ (we consider $\{1,2,3\}$ with the discrete topology).

Since $\mathbb{F}$ is not binary, we have $\{1,2\}^{+} \cap\{2,3\}^{+} \cap\{1,3\}^{+}=\emptyset$ by Theorem 3.5. Since $\{1,2\}^{+} \cap\{1,3\}^{+}$is a closed subset in $F\{1,2,3\}$, there exist $\varepsilon>0, c_{1}, \ldots, c_{k} \in \mathbb{R}$ and functions $\psi_{1}, \ldots, \psi_{k} \in C(\{1,2,3\})$ such that $\psi_{i}(2)=\psi_{i}(3)=c_{i}$ and for each $v \in$ $\{1,2\}^{+} \cap\{1,3\}^{+}$there exists $i \in\{1, \ldots, k\}$ such that $\left|v\left(\psi_{i}\right)-c_{i}\right| \geq \varepsilon$.

Let us remark that for each $\alpha, \beta \in \Lambda$ and $i \in\{1, \ldots, k\}$ we have $\psi_{i} \circ h_{\alpha}=\psi_{i} \circ h_{\beta}$. Hence for each $i \in\{1, \ldots, k\}$ there exists $\varphi_{i} \in C(Y)$ such that $\varphi_{i}=\psi_{i} \circ h_{\alpha}$ for each $\alpha \in \Lambda$. Put $D_{i}=\left\{\mu \in F Y|| \mu\left(\varphi_{i}\right)-c_{i} \mid \geq \varepsilon\right\}$ and $V=F X \backslash\left(\cup_{i=1}^{k} F f\left(D_{i}\right)\right)$.

Consider any $\alpha \in \Lambda$ and $v \in A_{\alpha}^{+} \cap B_{\alpha}^{+}$. Since $F$ preserves epimorphisms, there exists $\mu \in$ $F Y$ such that $F f(\mu)=v$. Then we have $\mu \in\left(f^{-1}\left(A_{\alpha}\right)\right)^{+} \cap\left(f^{-1}\left(B_{\alpha}\right)\right)^{+}$by Lemma 3.3. Since $f^{-1}\left(A_{\alpha}\right) \subset Y_{1} \cup Y_{2}^{\alpha}$ and $f^{-1}\left(B_{\alpha}\right) \subset Y_{1} \cup Y_{3}^{\alpha}$, we obtain $\mu \in\left(Y_{1} \cup Y_{2}^{\alpha}\right)^{+} \cap$ $\left(Y_{1} \cup Y_{3}^{\alpha}\right)^{+}$. Hence $F h_{\alpha}(\mu) \in\{1,2\}^{+} \cap\{1,3\}^{+}$by Lemma 3.2. Then there exists $i \in$ $\{1, \ldots, k\}$ such that $\varepsilon \leq\left|F h_{\alpha}(\mu)\left(\psi_{i}\right)-c_{i}\right|=\left|\mu\left(\psi_{i} \circ h_{\alpha}\right)-c_{i}\right|=\left|\mu\left(\varphi_{i}\right)-c_{i}\right|$ and $v \in F f\left(D_{i}\right) \subset F X \backslash V$.

Now, consider any $v \in C^{+}$and any $\mu \in F Y$ such that $F f(\mu)=\nu$. By Lemma 3.3, $\mu \in\left(f^{-1}(C)\right)^{+} \subset\left(Y_{2}^{\alpha} \cup Y_{3}^{\alpha}\right)^{+}$for each $\alpha \in \Lambda$. By Lemma 3.2 $F h_{\alpha}(\mu) \in\{2,3\}^{+}$. Hence $c_{i}=F h_{\alpha}(\mu)\left(\psi_{i}\right)=\mu\left(\varphi_{i}\right)$ for each $i \in\{1, \ldots, k\}$ and $v \in F X \backslash\left(\cup_{i=1}^{k} F f\left(D_{i}\right)\right)=V$. The lemma is proved.

We will need certain information about inverse systems and spectral methods developed by Shchepin to study topology of non-metrizable compacta (see [19] for more details). Suppose that $A$ is a partially ordered up-directed set. A subset $B$ of $A$ is said to be closed if for each chain $C$ in $B$ we have $\sup C \in B$ whenever $\sup C$ exists in $A$. An up-directed set $A$ is called $\tau$-complete (where $\tau$ is an infinite cardinal) if for every chain $B$ in $A$ with $|B| \leq \tau$ there exists an element $\sup B$ in $A$. A continuous inverse system $\mathcal{S}=\left\{X_{\alpha}, p_{\alpha}^{\beta}, A\right\}$ is called a $\tau$-system if its index set $A$ is $\tau$-complete and if the spaces $X_{\alpha}, \alpha \in A$, have 
weight $\leq \tau$. By $\lim \mathcal{S}$ and by $p_{\alpha}, \alpha \in A$, we denote the limit space of our system and the natural limit projections from $\lim \mathcal{S}$ to $X_{\alpha}$. The system $\mathcal{S}=\left\{X_{\alpha}, p_{\alpha}^{\beta}, A\right\}$ with $X=\lim \mathcal{S}$ is called factorizing if for each continuous function $f: X \rightarrow \mathbb{R}$ there exist $\alpha \in A$ and a continuous function $f_{\alpha}: X_{\alpha} \rightarrow \mathbb{R}$ such that $f_{\alpha} \circ p_{\alpha}=f$. Each $\tau$-system such that all $X_{\alpha}$ are compacta is factorizing [19, Proposition 1.6].

We will use the following fact, which is a particular case of Shchepin Spectral Theorem [19]. Let $\mathcal{S}=\left\{X_{\alpha}, p_{\alpha}^{\beta}, A\right\}$ and $\mathcal{L}=\left\{Y_{\alpha}, \varrho_{\alpha}^{\beta}, A\right\}$ be two $\tau$-systems where all $X_{\alpha}$ and $Y_{\alpha}$ are compacta and $\tau$ is an infinite cardinal. Then for each continuous map $f: X \rightarrow Y$ between the corresponding limit spaces $X=\lim \mathcal{S}, Y=\lim \mathcal{L}$ there exists a cofinal closed subset $B \subset A$ and a family $\left\{f_{\beta}: X_{\beta} \rightarrow Y_{\beta}\right\}_{\beta \in B}$ of continuous maps such that $f_{\beta} \circ p_{\beta}=$ $\varrho_{\beta} \circ f$ for each $\beta \in B$. Moreover, if $f$ is a homeomorphism, we can assume that all $f_{\beta}$ are homeomorphisms.

A functor $F$ is called continuous if it preserves the limits of inverse systems. Let us remark that the probability measure functor $P$ is continuous, preserves weights of infinite compacta and epimorphisms (see [6] for more information about the functor $P$ ).

A compactum $X$ is called an absolute extensor in the class of 0 -dimensional compacta (shortly $\mathrm{AE}(0)$ ) if for any 0 -dimensional compact space $Z$, any closed subspace $Y$ of $Z$ and any continuous map $\Phi: Y \rightarrow X$ there exists a continuous map $G: Z \rightarrow X$ such that $G \mid Y=\Phi$.

Theorem 4.2 Let $\mathbb{F}=(F, \eta, \mu)$ be an L-monad such that $F$ is continuous, preserves weights of infinite compacta, epimorphisms and one-point preimages. If $F\left(P D^{A}\right) \in A E(0)$ for a set $A$ with $|A| \geq \omega_{2}$, then the monad $\mathbb{F}$ is binary.

Proof Let $F\left(P D^{A}\right) \in A E(0)$ for a set $A$ with $|A| \geq \omega_{2}$. Firstly we introduce some nota-

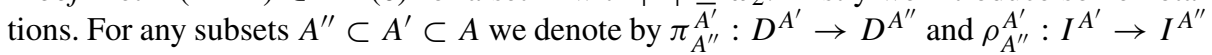
the natural projections. We also put $p_{A^{\prime \prime}}^{A^{\prime}}=F\left(P \pi_{A^{\prime \prime}}^{A^{\prime}}\right): F\left(P D^{A^{\prime}}\right) \rightarrow F\left(P D^{A^{\prime \prime}}\right)$. Let us write $\pi_{A^{\prime \prime}}, \rho_{A^{\prime \prime}}$ and $p_{A^{\prime \prime}}$ for $\pi_{A^{\prime \prime}}^{A}, \rho_{A^{\prime \prime}}^{A}$ and $p_{A^{\prime \prime}}^{A}$ respectively.

Consider any embedding $i: F\left(P D^{A}\right) \rightarrow I^{A}$. Put $A_{1}=\bigsqcup_{\alpha \in A} N_{\alpha}$ where each $N_{\alpha}=\mathbb{N}$. Evidently $\left|A_{1}\right|=|A|$ and $D^{A_{1}} \cong \prod_{\alpha \in A} D^{N_{\alpha}}$, thus we shall identify these two spaces. Let $s_{\alpha}: D^{N_{\alpha}} \rightarrow I$ be a continuous surjective map. Define a map $k: D^{A_{1}} \rightarrow I^{A}$ as follows: $k=\prod_{\alpha \in A} s_{\alpha}$. Since $F\left(P D^{A}\right) \in A E(0)$, there exists a map $f: D^{A_{1}} \rightarrow F\left(P D^{A}\right)$ such that $i \circ f(z)=k(z)$ for each $z \in k^{-1}\left(i\left(F\left(P D^{A}\right)\right)\right.$.

Let $\mathcal{A}=\left\{C \subset A \mid\right.$ card $\left.C \leq \omega_{1}\right\}$ and put $C_{1}=\bigsqcup_{\alpha \in C} N_{\alpha}$ for each $C \in \mathcal{A}$. By $k_{C}$ : $D^{C_{1}} \rightarrow I^{C}$ we denote the map $k=\prod_{\alpha \in C} s_{\alpha}$. Consider the following three inverse systems $\left\{I^{C}, \rho_{B}^{C}, \mathcal{A}\right\},\left\{D^{C_{1}}, \pi_{B_{1}}^{C_{1}}, \mathcal{A}\right\}$ and $\left\{F\left(P D^{C}\right), p_{B}^{C}, \mathcal{A}\right\}$ over the directed set $\mathcal{A}$. Evidently, the first two systems are $\omega_{1}$-systems with the limit spaces $I^{A}$ and $D^{A_{1}}$ respectively. Since the functors $F$ and $P$ are continuous and preserve weights of infinite compacta, the third system is an $\omega_{1}$-system with the limit space $F\left(P D^{A}\right)$.

Consider the continuous map $i: F\left(P D^{A}\right) \rightarrow I^{A}$. Consider the subfamily $\mathcal{B}_{1} \subset \mathcal{A}$ consisting of all $B \in \mathcal{A}$ for which there exists a continuous map $i_{B}: F\left(P D^{B}\right) \rightarrow I^{B}$ such that the diagram

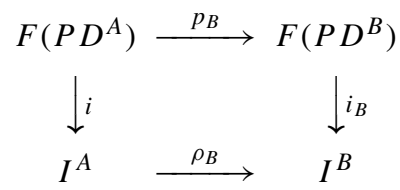


is commutative. Then by Shchepin Theorem discussed before $\mathcal{B}_{1}$ is a cofinal closed subfamily of $\mathcal{A}$. Moreover, considering the homeomorphism $i^{\prime}: F\left(P D^{A}\right) \rightarrow i^{\prime}\left(F\left(P D^{A}\right)\right)$ and the $\omega_{1}$-system $\left\{\rho_{C}\left(i^{\prime}\left(F\left(P D^{A}\right)\right)\right), \rho_{B}^{C} \mid \rho_{C}\left(i^{\prime}\left(F\left(P D^{A}\right)\right)\right), \mathcal{A}\right\}$ we can state that all $i_{B}$ are topological embeddings.

Now consider the continuous map $f: D^{A_{1}} \rightarrow F\left(P D^{A}\right)$ and the subfamily $\mathcal{B}_{2} \subset \mathcal{A}$ consisting of all $B \in \mathcal{A}$ for which there exists a continuous map $f: D^{B_{1}} \rightarrow F\left(P D^{B}\right)$ such that the diagram

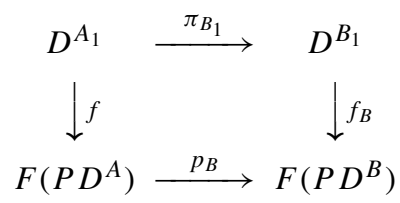

is commutative. As before $\mathcal{B}_{2}$ is a cofinal closed subfamily of $\mathcal{A}$.

Then the family $\mathcal{B}=\mathcal{B}_{1} \cap \mathcal{B}_{2}$ is cofinal too. Moreover, following either the proof of Lemma 2 from [1] or the proof of Shchepin theorem [19] (both are based on so-called spectral search which makes countable number of steps) we can assume that for each infinite set $A \in \mathcal{A}$ there exists $B \in \mathcal{B}$ such that $A \subset B$ and $|A|=|B|$. We note that for each $B \in \mathcal{B}$ the diagram

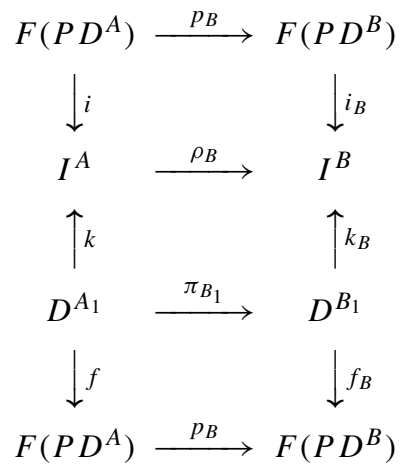

is commutative. It is easy to check that we have $i_{B} \circ f_{B}(z)=k_{B}(z)$ for each $z \in$ $k_{B}^{-1}\left(i_{B}\left(F\left(P D^{A}\right)\right)\right.$.

Choose a set $B \in \mathcal{B}$ such that $|B|=\omega_{1}$. Choose any point $a \in A \backslash B$ and countable infinite subset $F \subset B$. Then there exists a countable set $E \in \mathcal{B}$ such that $\{a\} \cup F \subset E$. Then we have $B \backslash E \neq \emptyset \neq E \backslash B$ and $B \cap E=C \in \mathcal{B}$ where $C$ is an infinite countable set. Put $T=\left\{(\mu, v) \in F\left(P D^{B}\right) \times F\left(P D^{E}\right) \mid p_{C}^{B}(\mu)=p_{C}^{E}(v)\right\}$. For each $(\mu, v) \in T$ we have $\rho_{C}^{B} \circ i_{B}(\mu)=i_{C} \circ p_{C}^{B}(\mu)=i_{C} \circ p_{C}^{E}(\nu)=\rho_{C}^{E} \circ i_{E}(\nu)$. So, there is a (uniquely determined) element $z$ of $I^{B \cup E}$ such that $\rho_{B}^{B \cup E}(z)=i_{B}(\mu)$ and $\rho_{E}^{B \cup E}(z)=i_{E}(v)$. We can define a map $j: T \rightarrow I^{B \cup E}$ by the formula $j(\mu, v)=z$. It is easy to check that the map $j$ is an embedding.

Define a continuous map $\sigma: D^{B_{1} \cup E_{1}} \rightarrow F\left(P D^{B}\right) \times F\left(P D^{E}\right)$ by the formula $\sigma=$ $\left(f_{B} \circ \pi_{B_{1}}^{B_{1} \cup E_{1}}, f_{E} \circ \pi_{E_{1}}^{B_{1} \cup E_{1}}\right)$. Then for each $t \in D^{B_{1} \cup E_{1}}$ we have $p_{C}^{B} \circ f_{B} \circ \pi_{B_{1}}^{B_{1} \cup E_{1}}(t)=$ $f_{C} \circ \pi_{C_{1}}^{B_{1}} \circ \pi_{B_{1}}^{B_{1} \cup E_{1}}(t)=f_{C} \circ \pi_{C_{1}}^{B_{1} \cup E_{1}}(t)=f_{C} \circ \pi_{C_{1}}^{E_{1}} \circ \pi_{E_{1}}^{B_{1} \cup E_{1}}(t)=p_{C}^{E} \circ f_{E} \circ \pi_{E_{1}}^{B_{1} \cup E_{1}}(t)$. Hence $\sigma(t) \in T$. 
Define a continuous map $p: F\left(P D^{A}\right) \rightarrow T$ by the formula $p=\left(p_{B}, p_{E}\right)$. We obtain a commutative diagram:

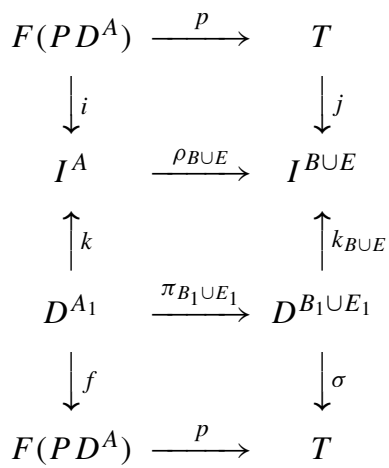

It is easy to check that we have $j \circ \sigma(z)=k_{B \cup E}(z)$ for each $z \in k_{B \cup E}^{-1}(j(T))$.

Let us show that $p$ is an open map. Consider any $v \in F\left(P D^{A}\right)$ and $K \subset T$ such that $p(v) \in \operatorname{cl} K$. We shall prove that $v \in \operatorname{cl}\left(p^{-1}(K)\right)$. Let $\left(d_{\alpha}\right)$ be a net in $K$ such that $\lim d_{\alpha}=$ $p(v)$. Then the net $\left(j\left(d_{\alpha}\right)\right)$ converges to $j(p(v))$. Since the last diagram is commutative, we have $\rho_{B \cup E}(i(v))=j(p(v))$. Choose $t_{\alpha} \in D^{B_{1} \cup E_{1}}$ such that $k_{B \cup E}\left(t_{\alpha}\right)=j\left(d_{\alpha}\right)$ for each $\alpha$. Since $D^{B_{1} \cup E_{1}}$ is compact, we can assume that $t_{\alpha}$ converges to some $t \in D^{B_{1} \cup E_{1}}$. Then $k_{B \cup E}(t)=j(p(v))=\rho_{B \cup E}(i(v))$ and we can choose $z \in D^{A_{1}}$ such that $k(z)=i(v)$ and $\pi_{B_{1} \cup E_{1}}(z)=t$. Then $f(z)=i^{-1}(k(z))=v$. Since the map $\pi_{B_{1} \cup E_{1}}$ is open, we can assume that there exists a net $\left(z_{\alpha}\right)$ which converges to $z$ and $\pi_{B_{1} \cup E_{1}}\left(z_{\alpha}\right)=t_{\alpha}$ for each $\alpha$. Put $v_{\alpha}=f\left(z_{\alpha}\right)$. Then the net $v_{\alpha}$ converges to $v$. Since the last diagram is commutative, we have $p\left(v_{\alpha}\right)=\sigma\left(t_{\alpha}\right)$. Since $k_{B \cup E}\left(t_{\alpha}\right)=j\left(d_{\alpha}\right)$, we have $\sigma\left(t_{\alpha}\right)=j^{-1}\left(k_{B \cup E}\left(t_{\alpha}\right)\right)=d_{\alpha}$. Hence $\operatorname{cl}\left(p^{-1}(K)\right) \supset \operatorname{cl}\left\{v_{\alpha}\right\} \ni v$ and $p$ is open.

Let us remark that the openness of the map $p$ implies the openness of the map $p_{1}$ : $F P\left(D^{B \cup E}\right) \rightarrow T$ defined as follows: $p_{1}=\left(p_{B}^{B \cup E}, p_{E}^{B \cup E}\right)$.

Choose an accumulation point $s_{0}$ of $D^{C}$, distinct points $r_{0}, r_{1} \in D^{B \backslash C}$ and $t_{0}, t_{1} \in D^{E \backslash C}$. Define a measure $\lambda \in P\left(D^{B \cup E}\right)=P\left(D^{B \backslash C} \times D^{C} \times D^{E \backslash C}\right)$ as follows:

$$
\lambda=\frac{1}{2}\left(\delta\left(r_{0}, s_{0}, t_{0}\right)+\delta\left(r_{1}, s_{0}, t_{1}\right)\right) .
$$

Choose disjoint closed neighborhoods $U_{0}, U_{1}$ of $r_{0}, r_{1}$ in $D^{B \backslash C}$ and $W_{0}, W_{1}$ of $t_{0}, t_{1}$ in $D^{E \backslash C}$, and let $V$ be the closed neighborhood of $\lambda$ in $P\left(D^{B \cup E}\right)$ defined by

$$
V=\left\{\lambda^{\prime} \in P\left(D^{B \cup E}\right) \mid \lambda^{\prime}\left(U_{0} \times D^{C} \times W_{0} \cup U_{1} \times D^{C} \times W_{1}\right) \geq \frac{1}{2}\right\} .
$$

Define the map $\gamma: P\left(D^{B \cup E}\right) \rightarrow Q=\left\{(\mu, \nu) \in P D^{B} \times P D^{E} \mid P\left(\pi_{C}^{B}\right)(\mu)=\right.$ $\left.P\left(\pi_{C}^{E}\right)(\nu)\right\}$ by $\gamma=\left(P\left(\pi_{B}^{B \cup E}\right), P\left(\pi_{E}^{B \cup E}\right)\right)$. Now $\gamma(\lambda)=(\mu, \nu)$, where

$$
\mu=\frac{1}{2}\left(\delta\left(r_{0}, s_{0}\right)+\delta\left(r_{1}, s_{0}\right)\right)
$$

and

For any point $s \in D^{C} \backslash\left\{s_{0}\right\}$ put

$$
v=\frac{1}{2}\left(\delta\left(s_{0}, t_{0}\right)+\delta\left(s_{0}, t_{1}\right)\right) .
$$

$$
\mu_{s}=\frac{1}{2}\left(\delta\left(r_{0}, s_{0}\right)+\delta\left(r_{1}, s\right)\right)
$$


and

$$
v_{s}=\frac{1}{2}\left(\delta\left(s, t_{0}\right)+\delta\left(s_{0}, t_{1}\right)\right) .
$$

Since $s_{0}$ is an accumulation point, we have $(\mu, v) \in \operatorname{cl}\left\{\left(\mu_{s}, v_{s}\right) \mid s \in D^{C} \backslash\left\{s_{0}\right\}\right\}$. Consider any $\lambda_{s} \in P\left(D^{B \cup E}\right)$ for which $\gamma\left(\lambda_{s}\right)=\left(\mu_{s}, v_{s}\right)$. Since $P\left(\pi_{B}^{B \cup E}\right)\left(\lambda_{s}\right)=\frac{1}{2}\left(\delta\left(r_{0}, s_{0}\right)+\right.$ $\left.\delta\left(r_{1}, s\right)\right)$, we have $\lambda_{s}=\frac{1}{2}\left(\lambda_{s}^{1}+\lambda_{s}^{2}\right)$ where $\lambda_{s}^{1} \in P\left(\left\{r_{0}\right\} \times\left\{s_{0}\right\} \times D^{E \backslash C}\right)$ and $\lambda_{s}^{2} \in P\left(\left\{r_{1}\right\} \times\right.$ $\left.\{s\} \times D^{E \backslash C}\right)$. Since $P\left(\pi_{E}^{B \cup E}\right)\left(\lambda_{s}\right)=\frac{1}{2}\left(\delta\left(s, t_{0}\right)+\delta\left(s_{0}, t_{1}\right)\right)$, we have $\lambda_{s}^{1} \in P\left(\left\{r_{0}\right\} \times\left\{s_{0}\right\} \times\right.$ $\left.\left\{t_{1}\right\}\right)$ and $\lambda_{s}^{2} \in P\left(\left\{r_{1}\right\} \times\{s\} \times\left\{t_{0}\right\}\right)$. Thus

$$
\lambda_{s}=\frac{1}{2}\left(\delta\left(r_{0}, s_{0}, t_{1}\right)+\delta\left(r_{1}, s, t_{0}\right)\right),
$$

which is not in $V$. Hence $\left(P\left(\pi_{B}^{B \cup E}\right)\right)^{-1}\left(\mu_{s}\right) \cap\left(P\left(\pi_{E}^{B \cup E}\right)\right)^{-1}\left(v_{s}\right) \cap V=\emptyset$ for each $s \in D^{C} \backslash\left\{s_{0}\right\}$.

Put $H_{s}=\left(P\left(\pi_{B}^{B \cup E}\right)\right)^{-1}\left(\mu_{s}\right)$ and $G_{s}=\left(P\left(\pi_{E}^{B \cup E}\right)\right)^{-1}\left(v_{s}\right)$. Then we have $H_{s}^{+} \cap G_{s}^{+}=$ $p_{1}^{-1}\left(\eta P D^{B}\left(\mu_{s}\right), \eta P D^{E}\left(v_{s}\right)\right)$ by Corollary 3.4. Since $p_{1}$ is open, we have $\eta P D^{B \cup E}(\lambda) \in$ $\operatorname{cl}\left(\cup\left\{H_{s}^{+} \cap G_{s}^{+} \mid s \in D^{C} \backslash\left\{s_{0}\right\}\right\}\right)$. Hence $\mathbb{F}$ is binary by Lemma 4.1 and the theorem is proved.

Open Access This article is distributed under the terms of the Creative Commons Attribution 4.0 International License (http://creativecommons.org/licenses/by/4.0/), which permits unrestricted use, distribution, and reproduction in any medium, provided you give appropriate credit to the original author(s) and the source, provide a link to the Creative Commons license, and indicate if changes were made.

\section{References}

1. Ditor, S., Haydon, R.: On absolute retracts, $P(S)$ and complemented subspaces of $C\left(D^{\omega_{1}}\right)$. Studia Math. 56, 243-251 (1976)

2. Ditor, S., Eifler, L.: Some open mapping theorems for measures. Trans. Amer. Math. Soc. 164, 287-293 (1972)

3. Eilenberg, S., Moore, J.: Adjoint functors and triples. Ill. J. Math. 9, 381-389 (1965)

4. Engelking, R.: Dimension theory. Warszawa (1978)

5. Fedorchuk: On softness of maps of subset in locally convex spaces. Uspekhi Mat. Nauk 45, 221-222 (1990)

6. Fedorchuk, V.V.: Probability measures in topology. Uspekhi Mat. Nauk 46, 41-80 (1991)

7. de Groot, J.: Supercompactness and Superextension. Contributions to extension theory of topological structures. Deuche Verlag der Wissenschaften, eds., Berlin (1967)

8. Ivanov, A.V.: Solution of van Mill's problem on the characterization of compacta, the superextensions of which are absolute retracts. Dokl. Akad. Nauk SSSR 262, 526-528 (1982)

9. Ivanov, A.V.: Superextensions of openly generated compacta. Dokl. Akad. Nauk SSSR 259, 275-278 (1981)

10. McLane, S.: Categories for Working Matematicians. Springer (1976)

11. Michael, E.: Some extension theorems for continuous functions. Pacific J. Math. 3, 789-806 (1953)

12. Radul, T., Zarichnyi, M.: Monads in the category of compacta. Uspekhi Mat. Nauk 50, 83-106 (1995)

13. Radul, T.: Convexities generated by L-monads. Appl. Categ. Struct. 19, 729-739 (2011)

14. Radul, T.: On functional representations of Lawson monads. Appl. Categ. Struct. 9, 457-463 (2001)

15. Radul, T.: On strongly Lawson and I-Lawson monads. Boletin de Matematicas 6, 69-75 (1999)

16. Radul, T.: Monad of hyperspaces of inclusion and its algebras. Ukr. Mat. Zh. 42, 806-811 (1990)

17. Swirszcz, T.: Monadic functors and convexity. Bull. Pol. Acad. Sci. 22, 39-42 (1970)

18. Teleiko, A., Zarichnyi, M.: Categorical Topology of Compact Hausdorff Spaces. VNTL Publishers, Lviv (1999)

19. Shchepin, E.V.: Functors and uncountable powers of compacta. Uspekhi Mat. Nauk 36, 3-62 (1981)

20. Shchepin, E.V.: $\kappa$-metrizable spaces. Izv. Akad. Nauk SSSR Ser. Mat. 43, 442-478 (1979)

21. van de Vel, M.: Theory of convex structures. North-Holland (1993)

22. Zarichnyi, M.: Spaces and mappings of idempotent measures. Izv. Ross. Akad. Nauk Ser. Mat. 74, 45-64 (2010) 